\title{
Fractional Domination in Graphs
}

\author{
D.K.Thakkar \\ Department of Mathematics, \\ Saurashtra University Campus, \\ University Road, Rajkot, India \\ dkthakkarl@yahoo.co.in
}

\author{
S.M.Badiyani \\ V.V.P Engineering College, \\ Kalawad Road, Virda Vagdi, \\ Rajkot, India \\ sankycolors@gmail.com
}

\begin{abstract}
In this paper we consider the effect of removing a vertex from a graph $G$ on the Fractional Domination Number of the graph. In particular we prove a necessary and sufficient condition under which the Fractional Domination Number of the given graph increases. We also prove that if the Fractional Domination Number decreases then it decreases by atmost 1.
\end{abstract}

Keywords: Dominating Set, Minimal Dominating Set, Minimum Dominating Set, Domination Number, Fractional Dominating Function, Minimal Fractional Dominating Function, Minimum Fractional Dominating Function, Fractional Domination Number.

AMS Mathematics Subject Classification (2010): 05C69

\section{INTRODUCTION}

Domination Number and related parameters have been studied by many authors and many research papers have been published related to Domination and its variants. The well-known books $[8,9]$ contains many such variants and related results. Several variants and their parameters have their Fractional Versions. Fractional Domination, Fractional Vertex Covering, Fractional Coloring are some of them. They provide generalizations of above variants and the values of related parameters are in general Fractions.

In this paper we consider one such concept called Fractional Domination. The introduction of this concept can be found in $[8,9]$. To be more precious a Dominating Set gives rise to a Dominating Function on the vertex set and vice-versa. The weight of a function replaces the cardinality of a set and vice - versa. Similarly a Minimal Dominating Set and a Minimum Dominating Set give rise to a Minimal Dominating Function and a Minimum Dominating Function respectively.

The operation of removing a vertex from a graph may increase, decrease or does not change the Domination Number of the graph. There are necessary and sufficient conditions under which the Domination Number increases or decreases [1]. It may be interesting to know what happens in the case of Fractional Domination.

The purpose of this paper is to state and prove necessary and sufficient conditions under which the Fractional Domination Number increases. Also we prove that if the Fractional Domination Number decreases then it decreases by atmost 1 .

\section{Preliminaries and Notations}

Let $G$ be a graph. $V(G)$ will denote the vertex set of $G$ and $E(G)$ will denote the edge set of $G$. If $S$ is a subset of $V(G)$ then $|S|$ denotes the cardinality of $S$. If $f: V(G) \rightarrow R$ (the set of Real Numbers) is any function then for any subset $S$ of $V(G) f(S)=\sum_{x \in S} f(x)$. The Number $f(V(G))$ is called the weight of $f$ and it is denoted as $w(f)$. If $f, g: V(G) \rightarrow$ Rare functions then we say that $f \leq g$ if $f(x) \leq$ $g(x)$ for every $x \in V(G)$ and we say that $f<g$ if $f \leq g$ and for some $x f(x)<g(x)$. We say that a function $f: V(G) \rightarrow R$ is Minimal with respect to some property $p$ if

i. $f$ satisfies property $p$. 


\section{D.K.Thakkar \& S.M.Badiyani}

ii. $\quad$ If $g<f$ thengdoes not have property $p$.

Afunction $f: V(G) \rightarrow R$ is said to be Minimum with respect to some property $p$ if its weight is minimum among all the functions satisfying property $p$. In a similar way we can define a Maximal Functions (w.r.t $p$ ) and Maximum Functions (w.r.t $p$ ).

Let $v$ be any vertex of the graph $G$ then $N(v)\{u \in V(G) u$ is adjacent to $v$ and $N[v]=N(v) \cup$ $\{v\}$. If $N[v]=\{v\}$ then $v$ is called an isolated vertex of a graph. If $v$ is not an isolated vertex then it is called a non- isolated vertex.

In this paper we consider only those graphs which have finite vertex set and are simple.

Definition 2.1. [5]

A set $S$ subset of $V(G)$ in a graph $G$ is said to be a "Dominating Set" if every vertex $v \in V(G)-S$ is adjacent to some vertex of $S$.

Definition 2.2. [5]

A Dominating Set $S$ with minimum cardinality is said to be a "Minimum Dominating Set".

Definition 2.3. [5]

A Dominating Set $S$ is a "Minimal Dominating Set" if no proper subset $S_{l}$ of $S$ is a Dominating Set.

Note that every Minimum Dominating Set is a Minimal dominating Set.

Definition 2.4. [5]

The cardinality of a Minimum Dominating Set is called the "Domination Number" of a graph $G$ and it is denoted by $\gamma(G)$.

Definition 2.5. [2]

A function $f: V(G) \rightarrow\{0,1\}$ is called "Dominating Function" if $\forall v \in V(G), f(N[v]) \geq 1$.

Theorem 2.6. [8]

Let $S$ be a Dominating subset of a graph $G$. Then the characteristic function of $S$ is a Dominating Function.

Proof: Omitted.

\section{Theorem 2.7. [8]}

Let $f: V(G) \rightarrow\{0,1\}$ be a Dominating Function. Let $S=\{v \in V(G) / f(v)=1\}$. Then $S$ is a Dominating Set $\& f$ is the characteristic function of $S$.

Proof: Omitted.

Definition 2.8. [2]

A Dominating Function $f: V(G) \rightarrow\{0,1\}$ is called a "Minimum Dominating Function" if its weight is minimum among all the Dominating Functions defined on $V(G)$.

Theorem 2.9. [8]

If $S$ is a Minimum Dominating Set then its characteristic Function $f$ is a Minimum Dominating Function.

Proof: Omitted

Theorem 2.10. [8]

Suppose $f$ is a Minimum Dominating Function defined on $V(G)$ then the set $S=\{v \in V(G) / f(v)=$ $1\}$ is a Minimum Dominating Set.

Proof: Omitted

Definition 2.11. [2]

A Dominating Function $f: V(G) \rightarrow\{0,1\}$ is called a "Minimal Dominating Function" if $\nexists$ a Dominating Function $g$ such that $g<f$. 
Theorem 2.12. [8]

Suppose $f: V(G) \rightarrow\{0,1\}$ is a Dominating Function then $f$ is a Minimal Dominating Function if and only if the set $S=\{x \in V(G) / f(x)=1\}$ is a Minimal Dominating Set.

Proof: Omitted

Theorem 2.13. [9]

A Dominating Function $f: V(G) \rightarrow\{0,1\}$ is a Minimal Dominating Function if and only if for any vertex $v$ for which $f(v)=1$ there exists a vertex $u \in N[v]$ such that $f(N[u])=1$.

Proof: Omitted

We have proved that every Dominating Set gives rise to a Dominating Function \& Converse is also true. The co-domain of this Dominating Function is $\{0,1\}$.

This definition of Dominating Function motivates to define the concept of Fractional Dominating Function. This concept does not require a set for Domination.

Definition 2.14. [4]

A function $f: V(G) \rightarrow[0,1]$ is called a "Fractional Dominating Functions" if for every $v \in V(G), f(N[v]) \geq 1$.

Definition 2.15. [4]

A Fractional Dominating Function with minimum weight is called a "Minimum Fractional Dominating Function". The weight of a Minimum Fractional Dominating Function is calledFractional Domination Number of a graph $G$ and it is denoted by $\gamma_{f}\{G\}$.

Note 2.16.

It may be noted that Fractional Domination Number is less than or equal to the Domination Number of the graph $G$.

Example 2.17. [9]: If $\mathrm{G}$ is a $\mathrm{K}$-Regular Graph $(K \geq 1)$ has $\mathrm{n}$ - vertices then $f(V)=\frac{1}{k+1}$ for every $v \in V(G)$ gives a Minimum Fractional Dominating Function and the weight of this function

is

$$
\frac{n}{k+1} \text {. }
$$

\section{Theorem 2.18.}

A Fractional Dominating Function $f: V(G) \rightarrow[0,1]$ is Minimal if and only if for every vertex $v \in V(G)$ for which $f(v)>0$; there is a vertex $u \in N[v]$ such that $f(N[u])=1$.

Proof: Suppose $f$ is Minimal.

Let $v \in V(G)$ be such that $f(v)>0$. Suppose there does not exist $u \in N[v]$ such that $f(N[u])=1$. Then $f(N[u])>1$ for every $u \in N[v]$. In particular, $f(N[v])>1$.

Then we may observe that $f(v)>1-\sum_{\substack{w \in N[v] \\ w \neq v}} f(w)$

Consider $h(u)>1-\sum_{\substack{w \in N(v) \\ w \neq v}} f(w) ; \forall u \in N[v]$

Define a function $g$ as follows

$$
\begin{gathered}
g(x)=f(x), \text { if } x \neq v \\
g(v)=\max \{h(u) / u \in N[v]\}
\end{gathered}
$$

It is obvious that $g(v)<f(v)$ which means that $g<f$. 
Let $x \in V(G)$.

Suppose $x \notin N[v]$ then

$$
g(N[u])=\sum_{w \in N[x]} g(w)=\sum_{w \in N[x]} f(w) \geq 1
$$

As $f$ is a Fractional Dominating Function.

Suppose $x \in N[v]$ then

$$
\begin{aligned}
g(N[x]) & =g(v)+\sum_{\substack{w \in N[x] \\
w \neq v}} g(w) \\
& =g(v)+\sum_{\substack{w \in N[x] \\
w \neq v}} f(w) \\
& \geq h(x)+\sum_{\substack{w \in N[x] \\
w \neq v}} f(w) \\
& \geq 1-\sum_{\substack{w \in N[x] \\
w \neq v}} f(w)+\sum_{\substack{w \in N[x] \\
w \neq v}} f(w) \\
\Rightarrow g(N[x]) & \geq 1
\end{aligned}
$$

Thus $g$ is a Fractional Dominating Function such that $g<f$ which is a contradiction to the hypothesis that $f$ is Minimal.

Thus there must be vertex $u \in N[v]$ such that $f(N[u])=1$, whenever $f(v)>0$.

Conversely, Suppose for $v$, for which $f(v)>0$ there exist a vertex $u \in N[v]$ such that $f(N[u])=1$.

Suppose $f$ is not a Minimal Fractional Dominating Function.

Therefore there is a Fractional Dominating Function $g$ such that $g<f$.

Therefore for some vertex $v \in V(G)$ we get $g(v)<f(v)$.

Let $u$ be a vertex such that $u \in N[v]$ such that $f(N[u])=1$. Then

$$
\begin{gathered}
g(N[u])=g(v)+\sum_{\substack{w \in N[u] \\
w \neq v}} g(w) \\
<f(v)+\sum_{\substack{w \in N[u] \\
w \neq v}} f(w) \\
=f(N[u])=1 \\
\Rightarrow \quad g(N[u])<1
\end{gathered}
$$

This contradicts the fact that $g$ is a Fractional Dominating Function.

So $f$ is a Minimal Fractional Dominating Function.

\section{Remark 2.19.}

Let $G$ be a graph and $v \in V(G)$. The subgraph $G-v$ is obtained by removing the vertex $v$ and all edges incident to $v$. The Fractional Domination Number of $G-v$ may be equal to or less than or greater than the Fractional Domination Number of the graph $G$.

If the Fractional Domination Number of a graph decreases, How Much it can decrease? The following Theorem provides an answer. 


\section{Theorem 2.20.}

Let $G$ be a graph and $v \in V(G)$.If $\gamma_{f}(G-v)<\gamma_{f}(G)$ then $\gamma_{f}(G)-1 \leq \gamma_{f}(G-v)<\gamma_{f}(G)$.

Proof: Suppose $\gamma_{f}(G-v)<\gamma_{f}(G)$

Let $g$ be a Minimum Fractional Dominating Function for $G-v$. Note that any function defined on $V(G)$ whose weight is equal to the weight of $g$ cannot be Fractional Dominating Function for $G$.

Suppose

$$
\sum_{w \in N(v)} g^{\prime}(w) \geq 1
$$

Define $g^{\prime}$ on $V(G)$ as follows

$$
\begin{gathered}
g^{\prime}(v)=0 ; \quad \text { and } \\
g^{\prime}(x)=g(x) ; \forall x \neq v
\end{gathered}
$$

Since

$$
\begin{aligned}
g^{\prime}(N[v]) & =g^{\prime}(v)+\sum_{w \in N(v)} g^{\prime}(w) \\
& =0+\sum_{w \in N(v)} g^{\prime}(w) \\
\Rightarrow & g^{\prime}(N[v]) \geq 1
\end{aligned}
$$

And $\operatorname{sog}$ ' is a Fractional Dominating Function for $\mathrm{G}$ with weight of $g^{\prime}=$ weight of $g$.

i.e. $W\left(g^{\prime}\right)=W(g)$.

This is a contradiction. So,

$$
\sum_{w \in N(v)} g^{\prime}(w)<1
$$

Now define $h$ on $V(G)$ as follows

$$
\begin{aligned}
& h(v)=1-\sum_{w \in N(v)} g(w) ; \text { and } \\
& h(x)=g(x) \quad ; \forall x \neq v
\end{aligned}
$$

Then

$$
\begin{aligned}
h(N[v]) & =h(v)+\sum_{w \in N(v)} g(w) \\
& =1-\sum_{w \in N(v)} g(w)+\sum_{w \in N(v)} g(w) \\
& \Rightarrow h(N[v])=1
\end{aligned}
$$

Thus $h$ is a Fractional Dominating Function on G.

Therefore $\gamma_{f}(G-v)<\gamma_{f}(G) \leq w(h) \leq w(g)+1$

$$
\begin{gathered}
\Rightarrow \gamma_{f}(G-v)<\gamma_{f}(G) \leq \gamma_{f}(G-v)+1 \\
\quad \Rightarrow \gamma_{f}(G)-\gamma_{f}(G-v) \leq 1 \\
\Rightarrow \gamma_{f}(G)-1 \leq \gamma_{f}(G-v)<\gamma_{f}(G)
\end{gathered}
$$

In the following theorem we will establish a necessary and sufficient condition under which the Fractional Domination Number of a graph increases. 


\section{Theorem 2.21.}

Let $\mathrm{G}$ be a graph and $v \in V(G)$, then $\gamma_{f}(G-v)>\gamma_{f}(G)$ if and only if the following three conditions hold:

i) $\quad v$ is not an isolated vertex of $G$.

ii) $\quad f(v) \neq 0$ For every Minimum Fractional Dominating function $f$.

iii) There is no function $g$ such that,

a) $\quad g$ is a Fractional Dominating function of $G-v$.

b) weight of $(g) \leq \gamma_{f}(G)$.

c) The support of $g$ is contained in $V(G)-N[v]$.

[If $h$ is a function from $V(G)$ to $[0,1]$ then support of $\quad h=S(h)=\{w \in V(G) / h(w)=1\}$ ]

Proof: First suppose that $\gamma_{f}(G-v)>\gamma_{f}(G)$

i) Suppose $v$ is an isolated vertex. Let $f$ be a Minimum Fractional Dominating Function of $G$. Since $N[v]=\{v\}$ and $f(N[v]) \geq 1, f(v)=1$.

Now define $h$ on $G$ - vas follows:

$$
h(w)=f(w) ; \forall w \in G-v
$$

Then obviously $h$ is a Fractional Dominating Function on $G-v$.

Therefore $\gamma_{f}(G-v) \leq$ weight of $h<$ weight of $f=\gamma_{f}(G)$.

$\Rightarrow \gamma_{f}(G-v) \leq \gamma_{f}(G)$; Which contradicts our hypothesis.

Thus $v$ cannot be an isolated vertex.

ii) Suppose there is a Minimum Fractional Dominating function $f$ such that $f(v)=0$. Now define $h$ on $G-v$ as follows:

$$
h(w)=f(w) ; \forall w \in G-v
$$

Then obviously $h$ is a Fractional Dominating Function on $G-\{v\}$.

Therefore $\gamma_{f}(G-v) \leq$ weight of $h \leq$ weight of $f=\gamma_{f}(G)$.

$\Rightarrow \gamma_{f}(G-v) \leq \gamma_{f}(G)$; This is again a Contradiction.

Thus $f(v) \neq 0$ for any Minimum Fractional Dominating function $f$ on $G$.

iii) Suppose there is a function $g$ satisfies condition $a), b$ ) and $c$ ) then

$$
\begin{aligned}
\gamma_{f}(G-v) & \leq \text { weight of } g \\
& \leq \gamma_{f}(G) \\
\Rightarrow \quad \gamma_{f}(G-v) & \leq \gamma_{f}(G) ; \text { This is a contradiction }
\end{aligned}
$$

Conversely, Suppose Condition i), ii) and iii) hold. First suppose $\gamma_{f}(G-v)=\gamma_{f}(G)$

Let $h$ be a Minimum Fractional Dominating Function of $G-v$.

Suppose $\sum_{w \in N(v)} h(w) \geq 1$

Now define $h^{\prime}$ on $V(G)$ as follows:

$$
\begin{gathered}
h^{\prime}(v)=0 ; \quad \text { and } \\
h^{\prime}(w)=h(w) ; \forall w \neq v
\end{gathered}
$$


Then $h^{\prime}$ is a Fractional Dominating Function on $\mathrm{G}$ and weight of $h^{\prime}=$ weight of $h \cdot \operatorname{Since} \gamma_{f}(G-v)=$ $\gamma_{f}(G) ; h^{\prime}$ must be a Minimum Fractional Dominating function for $G$.

Thus we have a Minimum Fractional Dominating function $h^{\prime}$ of such that $h^{\prime}(v)=0$; which contradicts ii) condition.

Suppose $\sum_{w \in N(v)} h(w)<1$

Then

a) his a Fractional Dominating function of $G-v$.

b) weight of $h \leq \gamma_{f}(G)$.

c) The support of $h$ is contained in $V(G)-N[v]$.

This contradicts iii) condition.

Thus $\gamma_{f}(G-v)=\gamma_{f}(G)$ is not possible.

Suppose $\gamma_{f}(G-v)<\gamma_{f}(G)$.

Let $g$ be a Minimum Fractional Dominating Function on $G-v$.

Suppose $\sum_{w \in N(v)} g(w) \geq 1$

Now define $g^{\prime}$ on $V(G)$ as follows

$$
\begin{gathered}
g^{\prime}(v)=0 ; \quad \text { and } \\
g^{\prime}(w)=g(w) ; \forall w \neq v
\end{gathered}
$$

Then $g^{\prime}$ is a Fractional Dominating Function on $G$.

Therefore $\gamma_{f}(G) \leq$ weight of $g^{\prime}=$ weight of $g=\gamma_{f}(G-v)$.

i.e. $\quad \gamma_{f}(G) \leq \gamma_{f}(G-v)$; Which isa contradiction.

Therefore $\sum_{w \in N(v)} g(w)<1$

This means that support of $g$ is subset of $V(G)-N[v], g$ is a Fractional Dominating Function on $G-v$ and weight of $g \leq \gamma_{f}(G)$.

This contradicts iii) condition.

Therefore $\gamma_{f}(G-v)<\gamma_{f}(G)$ is also not possible.

Thus It must be true that $\gamma_{f}(G-v)>\gamma_{f}(G)$.

Theorem 2.22.

Let $G$ be a graph and $v \in V(G)$. If $\gamma_{f}(G-v)<\gamma_{f}(G)$ then there is a Fractional Dominating Function $h$ defined on $G$ such that

1) $h(v) \neq 0$

2) $h(N[v])=1$

3) The restriction of $h$ on $G-v$ is a Minimum Fractional Dominating Function.

The function $h$ is minimal with respect to property 1), 2) and 3).

Proof: Let $g$ be a Minimum Fractional Dominating Function for the subgraph $G-v$. Then as proved in the above theorem $\sum_{w \in N(v)} g(w)<1$. 
Define $h$ on $G$ as follows

$$
\begin{array}{ll}
h(v)=1-\sum_{w \in N(v)} g(w) ; \text { and } \\
h(x)=g(x) \quad ; \forall x \neq v
\end{array}
$$

1) Obviously $h(v) \neq 0$

$$
\begin{array}{rlrl} 
& h(N[v]) & =h(v)+\sum_{w \in N(v)} g(w) \\
\Rightarrow \quad & h(N[v])=1-\sum_{w \in N(v)} g(w)+\sum_{w \in N(v)} g(w) \\
\Rightarrow \quad & h(N[v])=1
\end{array}
$$

Obviously $h$ is a Fractional Dominating Function on $G$ and $h(N[v])=1$.

3) The restriction of $h$ on $G-v$ is equal to $g$ which is a MinimumFractional Dominating Function on $G-v$.

Suppose $h^{\prime}$ is a Fractional Dominating Function on G which satisfies 1),2) and 3) and $h^{\prime}<h$.

Suppose $h^{\prime}(v)<h(v)$

$\operatorname{Then} h^{\prime}(v)<1-\sum_{w \in N(v)} g(w)$

$\Rightarrow \quad h^{\prime}(v)+\sum_{w \in N(v)} g(w)<1$

$h^{\prime}(N[v])=h^{\prime}(v)+\sum_{w \in N(v)} h^{\prime}(w)<h(v)+\sum_{w \in N(v)} h(w)=1$

Therefore $h^{\prime}(N[v])<1$ which is a contradiction; as $h^{\prime}$ is a Fractional Dominating Function.

Therefore $h^{\prime}(v)=h(v)$.

Therefore there is a vertex $x$ such that $x \neq v$ and $h^{\prime}(x)<h(x)=g(x)$.

Therefore when $h^{\prime}$ is restricted to $G-v, h^{\prime}<g$ and therefore $h^{\prime}$ is not a Fractional Dominating Function which is again a contradiction.

Therefore there is no function $h^{\prime}$ which satisfies property 1),2) and 3) and $h^{\prime}<h$.

Thus $h$ is Minimal with respect to property 1$), 2$ ) and 3 ).

\section{Concluding Remarks}

It seems difficult to establish a necessary and sufficient condition (or a set of conditions) under which the Fractional Domination Number decreases when a vertex is removed from the graph. The restriction of a Minimum Function on $G-v$ may not be a Fractional Dominating Function.

\section{REFERENCES}

[1] C. Berge "Theory of Graphs \& its Applications" Methuen, London, (1962).

[2] F.R.K. Chung Z Furedi, M.R.Garey \& R.L.Graham, One the Fractional Covering Number of Hypergraphs. SIAM J Discrete Math 1 45-49 (1988).

[3] J.E.Dunbar, S.T. Hedetniemi, M.A.Henning, and P.J.Slater. Signed domination in graphs. In Y.Alavi and A.J.Schwenk, editors, Graph Theory, Combinatorics, and Applications, Proc. $7^{\text {th }}$ Internat.Conf. Combinatorics, Graph Theory, Applications, Volume 1, pages 311-322.John Wiley \&sons, Inc.(1995).

[4] M.Farber, Domination, Independent Domination and duality in strongly chordal graphs, discrete Appl. Math. 7 115-130 (1986). 
[5] O. Ore "Theory of Graphs", Amer. Math. Soc. Collaq. Publ., 38 (Amer. Math. Soc., Providence, RI) (1962).

[6] S.M.Hedetniemi, S.T.Hedetniemi \& T.V.Wimer, Linear time resource allocation for trees. Technical Report URI-014, Dept. Mathematical Sciences, Clemson, Univ.(1987)

[7] S.T.Hedetniemi \& R.C.Laskar, editors. "Topics on Domination" Volume 48. North Holland, New York (1990).

[8] Teresa.W.Haynes, Stephen.T.Hedetniemi and Peter.J. Slater, "Fundamentals Of Domination In Graphs". (1998).

[9] Teresa.W.Haynes, Stephen.T.Hedetniemi and Peter.J. Slater, “ Domination In Graphs Advanced Topics"(1998).

\section{AUTHORS' BIOGRAPHY}

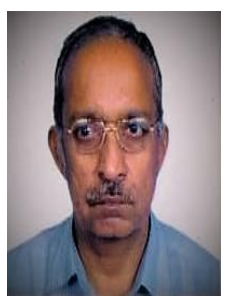

Dr.D.K.Thakkar, is in the Department of Mathematics of Saurashtra University, Rajkot. His areas of interest are Graph Theory, Topology and Discrete Mathematics. He has published over 45 research papers in various journals.

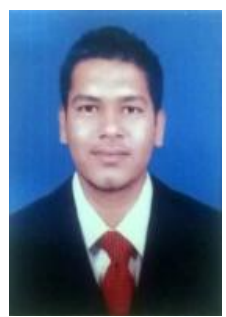

Mr S.M.Badiyani, is a young researcher student who is working in the area of Domination in graphs. He is working in V.V.P Engineering College Rajkot as a Lecturer. He like to work in challenging environment. 